\title{
Exodontia atraumática por meio da piezocirurgia em tratamento de erupção ectópica em seio maxilar: relato de caso
}

Atraumatic tooth extraction by means of piezosurgery in the treatment of ectopic eruption

in the maxillary sinus: case report

Extracción dentaria atraumática mediante piezocirugía en el tratamiento de la erupción ectópica en el seno maxilar: reporte de caso

Layla Louise de Amorim ROCHA ${ }^{1}$

Matheus Francisco Barros RODRIGUES ${ }^{\mathbf{1}}$

Iana Maria Gomes BARBOSA ${ }^{2}$

Rodrigo da França ACIOLY ${ }^{3}$

Daniel do Carmo CARVALHO ${ }^{3}$

Dennis Dinelly de SOUZA ${ }^{3}$

${ }^{1}$ Curso de Odontologia, Faculdade Cathedral, 69307-053 Boa Vista - RR, Brasil

${ }^{2}$ Cirurgiã-Dentista, Clínica Odontológica Privada, 69301-110 Boa Vista - RR, Brasil

${ }^{3}$ Departamento de Cirurgia e Traumatologia Bucomaxilofacial, Hospital da Criança Santo Antônio, 69308-160 Boa Vista - RR, Brasil

\begin{abstract}
Resumo
Erupção dentária ectópica pode resultar devido processo patológico, distúrbio do desenvolvimento ou atividade iatrogênica. Elementos dentário ectópicos podem ser encontrados em região de palato, região de seio maxilar, côndilo mandibular, processo coronóide, cavidades orbitais e nasais. A justificativa desse estudo dar-se pelo fato de que falha no tratamento de elementos dentários não irrompidos pode levar à reabsorção externa dos dentes adjacentes, problemas estéticos, arcos dentários encurtados e aumento da formação de cistos foliculares e infecções recorrentes. Nesse âmbito, o objetivo desse estudo é realizar um relato de caso de tratamento de elemento dentário ectópico em região de seio maxilar por meio da piezocirurgia. Paciente do sexo feminino, 16 anos, compareceu ao consultório odontológico com queixas álgicas. Ao exame clínico foi possível observar a ausência do elemento dentário 13. Nos exames de imagem foi possível observar uma erupção dentária ectópica em região de parede anterior de seio maxilar, com parte do elemento dentário incluso em seio. O tratamento instituído foi de remoção cirúrgica do dente por meio da técnica atraumática de piezocirurgia. O tratamento cirúrgico de um elemento impactado requer técnicas de osteotomia e odontossecção que, por si só, causam danos aos tecidos. No entanto, a cirurgia assistida por piezocirurgia é útil para minimizar os traumas na região. Pode-se concluir que a abordagem de remoção cirúrgica do elemento ectópico em seio maxilar foi satisfatória para resolução do quadro.
\end{abstract}

Descritores: Piezocirurgia; Seio Maxilar; Procedimentos Cirúrgicos Bucais.

\section{Abstract}

Ectopic tooth eruption can result due to pathological process, developmental disorder or iatrogenic activity. Ectopic dental elements can be found in the palate region, maxillary sinus region, mandibular condyle, coronoid process, orbital and nasal cavities. The justification for this study is due to the fact that failure to treat unerupted dental elements can lead to external resorption of adjacent teeth, aesthetic problems, shortened dental arches and increased formation of follicular cysts and recurrent infections. In this context, the objective of this study is to carry out a case report of treatment of an ectopic dental element in the maxillary sinus region by means of piezosurgery. Female patient, 16 years old, came to the dental office with pain complaints. On clinical examination, it was possible to observe the absence of dental element 13 . In imaging studies, it was possible to observe an ectopic dental eruption in the region of the anterior wall of the maxillary sinus, with part of the dental element included in the sinus. The treatment instituted was surgical removal of the tooth using the atraumatic technique of piezosurgery. Surgical treatment of an impacted element requires osteotomy and odontosection techniques that, by themselves, cause tissue damage. However, piezosurgery-assisted surgery is useful to minimize trauma to the region. It can be concluded that the approach of surgical removal of the ectopic element in the maxillary sinus was satisfactory for resolving the condition.

Descriptors: Piezosurgery; Maxillary Sinus; Oral Surgical Procedures.

\section{Resumen}

La erupción de un diente ectópico puede deberse a un proceso patológico, un trastorno del desarrollo o una actividad iatrogénica. Los elementos dentales ectópicos se pueden encontrar en la región del paladar, la región del seno maxilar, el cóndilo mandibular, la apófisis coronoides, las cavidades orbitaria y nasal. La justificación de este estudio se debe al hecho de que la falta de tratamiento de los elementos dentales no erupcionados puede conducir a la reabsorción externa de los dientes adyacentes, problemas estéticos, arcos dentales acortados y aumento de la formación de quistes foliculares e infecciones recurrentes. En este contexto, el objetivo de este estudio es realizar un reporte de caso de tratamiento de un elemento dentario ectópico en la región del seno maxilar mediante piezocirugía. Paciente mujer de 16 años que acude al consultorio odontológico con quejas de dolor. En el examen clínico se pudo observar la ausencia del elemento dentario 13. En los estudios de imagen se pudo observar una erupción dentaria ectópica en la región de la pared anterior del seno maxilar, con parte del elemento dentario incluido en el seno. El tratamiento instituido fue la extirpación quirúrgica del diente mediante la técnica atraumática de la piezocirugía. El tratamiento quirúrgico de un elemento impactado requiere técnicas de osteotomía y odontosección que, por sí mismas, causan daño tisular. Sin embargo, la cirugía asistida por piezocirugía es útil para minimizar el trauma en la región. Se puede concluir que el abordaje de la extirpación quirúrgica del elemento ectópico en el seno maxilar fue satisfactorio para resolver la afección.

Descriptores: Piezocirugía; Seno Maxilar; Procedimientos Quirúrgicos Orales.

INTRODUÇÃO

O canino superior é o dente mais frequentemente envolvido em impactação, com prevalência de 0,9 e $2,2 \%$ e em sua maioria estão posicionados ectopicamente ${ }^{1,2}$. Erupção dentária ectópica pode resultar devido processo patológico, distúrbio do desenvolvimento ou atividade iatrogênica ${ }^{3}$. Elementos dentários ectópicos podem ser encontrados em região de palato, região de seio maxilar, côndilo mandibular, processo coronóide, cavidades orbitais e nasais ${ }^{4}$. A erupção ectópica em seio maxilar pode apresentar-se como uma condição 
assintomática ou sintomática, a qual pode ser encontrar edema nasal, cefaleia, coriza e desvio da anatomia nasomaxilar ${ }^{5}$.

A falha em diagnosticar, gerenciar ou tratar casos de elementos dentários não irrompidos pode levar à reabsorção externa dos dentes adjacentes, problemas estéticos, desalinhamento dos dentes vizinhos, arcos dentários encurtados e aumento da formação de cistos foliculares e infecções recorrentes ${ }^{6,7}$. O tratamento instituído para os casos de inclusão dentária geralmente é cirúrgico com priorização da exodontia atraumática, por diminuir os traumas de natureza operatória ${ }^{6,7}$.

O uso da piezocirurgia permite realizar cortes ósseos mais finos e estáveis, além de facilitar a mínimo sangramento intraoperatória, proporcionando uma melhor visão da área cirúrgica ${ }^{6,9}$. Além de permitir a realização de osteotomias por meio de corte seletivo sem risco de necrose por superaquecimento ou dano neural ${ }^{9}$.

Esse estudo se justifica pelo fato de que falha no tratamento de elementos dentários não irrompidos pode levar à reabsorção externa dos dentes adjacentes, problemas estéticos, arcos dentários encurtados e aumento da formação de cistos foliculares e infecções recorrentes ${ }^{6,7}$. Nesse âmbito, o objetivo desse estudo é realizar um relato de caso de tratamento de elemento dentário ectópico em região de seio maxilar por meio da piezocirurgia.

\section{CASO CLÍNICO}

Paciente do sexo feminino, 16 anos, compareceu ao consultório odontológico com queixas álgicas. Ao exame clínico foi possível observar a ausência do elemento dentário 13, logo foi solicitado exame de radiografia panorâmica e tomografia computadorizada (Figuras 1 e 2). Nos exames de imagem foi possível observar uma erupção dentária ectópica em região de parede anterior de seio maxilar, com parte do elemento dentário incluso em seio. O tratamento instituído foi de remoção cirúrgica do dente por meio da técnica atraumática de piezocirurgia. A paciente foi submetido a cirurgia sob anestesia local com anestésico Articaína 4\% e sedação endovenosa, por sofrer ataques epilépticos. No ato operatório foi realizada a incisão em região vestibular com $3 \mathrm{~mm}$ de extensão, acima da linha mucogengival e com auxílio do piezoelétrico foi feito uma janela óssea em parede anterior de seio para acessar o dente (Figura 3) e posteriormente foi removido com auxílio de alavanca Heidbrink e Porta-agulha Mayo (Figura 4).

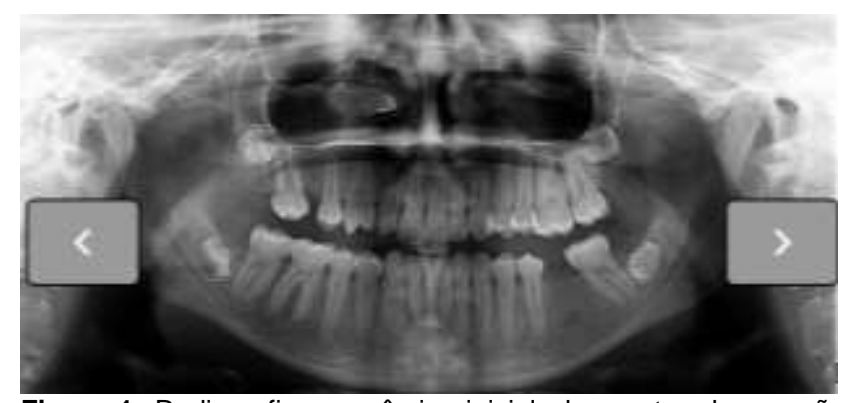

Figura 1: Radiografia panorâmica inicial, demonstrando erupção ectópica do elemento 13 em região de seio maxilar.

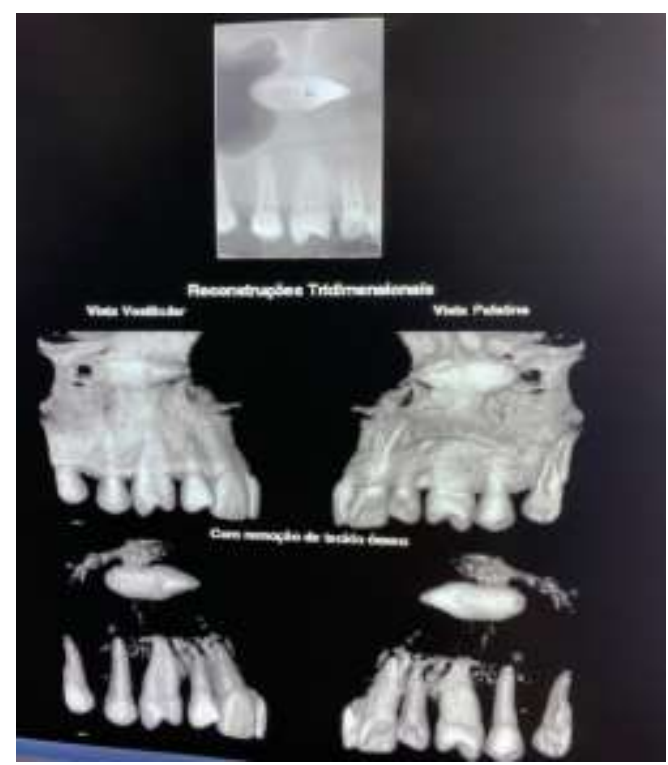

Figura 2: Tomografia computadorizada com reconstrução tridimensional em vistas vestibular e palatina.

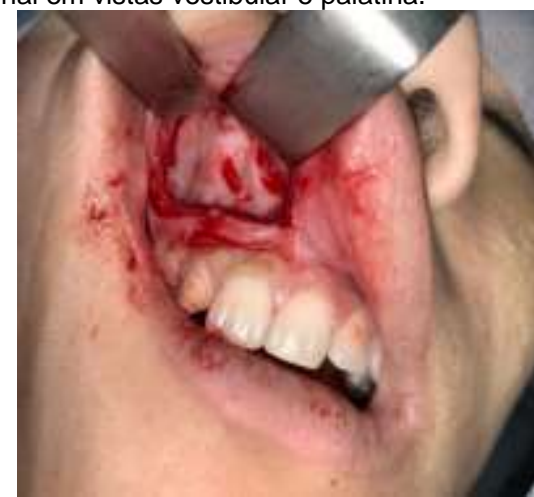

Figura 3: Acesso vestibular acima da linha mucogengival.

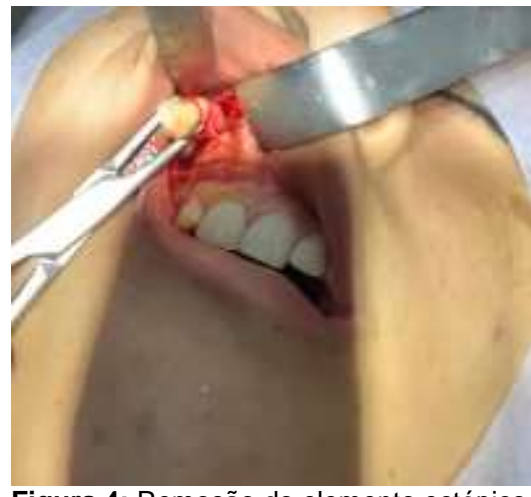

Figura 4: Remoção do elemento ectópico.

Por fim, foi reposicionado o tecido por meio de sutura contínua festonada. A medicação pós-operatória consistiu em Dipirona Sódica (500mg de 6 em 6 horas), Meloxican 
(7,5mg 12 em 12 horas) e Amoxicilina (500mg de 8 em 8 horas) No pós-operatório imediato 0 paciente não apresentou quadro de inflamação patológica ou infeccioso.

DISCUSSÃO

Exposição cirúrgica, tração ortodôntica, autotransplante e remoção cirúrgica podem apresentar-se como alternativa eficaz para tratamento de dentes não irrompidos ${ }^{10}$. No entanto, idade do paciente, o estágio de formação da raiz, a proximidade do dente ao local, a angulação do dente entre 0 e $15^{\circ}$ e a ausência de dilaceração da raiz podem interferir no tratamento ortodôntico ${ }^{6,11}$.

No caso clínico relatado o elemento dentário ectópico em região de seio maxilar inviabilizou o tratamento ortodôntico. Portanto, o tratamento instituído foi de remoção cirúrgica, levando em consideração fatores relacionados a profundidade de impactação, condição da raiz e a idade do paciente favorável a realização do procedimento.

O tratamento cirúrgico de um elemento impactado requer técnicas de osteotomia e odontossecção que, por si só, causam danos aos tecidos ${ }^{6,11}$. No entanto, a cirurgia assistida por piezocirurgia é útil para minimizar os traumas na região ${ }^{12,13}$. O uso de serras comuns ou brocas de alta velocidade aplica pressão e um certo grau de aquecimento ao osso e ao tecido mole adjacente ${ }^{14}$. O equipamento piezoelétrico resulta em aquecimento mínimo, diminuindo o risco de osteonecrose e garantindo a vitalidade dos osteócitos ${ }^{6,14}$.

\section{CONCLUSÃO}

Pode-se concluir que a abordagem de remoção cirúrgica do elemento ectópico em seio maxilar foi satisfatória para resolução do quadro. O paciente apresentou bom estado geral no pós-operatório, ausência de quadro inflamatório patológico ou infeccioso. A piezocirurgia foi a técnica preconizada para realização do procedimento, uma vez que apresenta mínimo trauma e diminui os riscos de necrose tecidual.

\section{REFERÊNCIAS}

1. Caprioglio A, Comaglio I, Siani L, Fastuca R. Effects of impaction severity of treated palatally displaced canines on periodontal outcomes: a retrospective study. Prog Orthod. 2019;20(1):5.

2. Daniels JS, Albakry I, Braimah RO, Samara MI. Ectopic eruption of canine into maxillary sinus with unusual clinical presentation: A case report and review of the literature. J Dent Res Rev 2019;6:52-5.
3. Almomen A, Alkhudair B, Alkhatib A, Alazzah G, Ali Z, Al Yaeesh I, AlOmairin A, Alshuaibi A, AlBahr A. Ectopic maxillary tooth as a cause of recurrent maxillary sinusitis: a case report and review of the literature. J Surg Case Rep. 2020;2020(9):rjaa334.

4. Liu J, Zhou M, Liu Q, He X, Wang N. Process of ectopic tooth formation in the maxillary sinus: follow-up observation of one case. J Int Med Res. 2019;47(12):6356-64.

5. Chagas Júnior OL, Moura LB, Sonego CL, de Farias EO, Giongo CC, Fonseca AA. Unusual Case of Sinusitis Related to Ectopic Teeth in the Maxillary Sinus Roof/Orbital Floor: A Report. Craniomaxillofac Trauma Reconstr. 2016;9(3):260-63.

6. Rodrigues MFB, de Amorim Rocha LL, da Franca Acioly R, da Rocha CCL, do Carmo Carvalho D. Piezosurgery-Assisted Surgical Treatment in Impacted Canine Transmigration. Case Rep Dent. 2020;2020:2687827.

7. Cavuoti S, Matarese G, Isola G, Abdolreza J, Femiano F, Perillo L. Combined orthodonticsurgical management of a transmigrated mandibular canine. Angle Orthod. 2016;86(4):681-91.

8. Büyükkurt MC, Tozoglu $S$, Aras $M H$, Yolcu U. Ectopic eruption of a maxillary third molar tooth in the maxillary sinus: a case report. J Contemp Dent Pract. 2005;6(3):104-10

9. Akçay H, Tatar B, Kuru K, Gözlüklü Ö, Ulu M. Bone Flap Technique With Piezosurgery for Impacted Teeth Extraction and Bone Cysts Removal Without Additional Fixation. J Craniofac Surg. 2019;30(1):e21-e24.

10. Ismail $M Q$, Lauridsen $E$, Andreasen JO, Hermann NV. Ectopic eruption of the second premolar: an analysis of four different treatment approaches. Eur Arch Paediatr Dent. 2020;21(1):119-27.

11. Rocha LLA, Rodrigues MFB, Barbosa IMG, Acioly RLX, Rocha CCL, Acioly RF et al. Atraumatic Surgical Removal of Impacted Deciduous and Premolar: Case Report. Oral Health Awareness.2020; 1(1)

12. Kumar MPS. Newer methods of extraction of teeth. Int J Pharm Bio Sci. 2015;6(3):679-85.

13. Rocha LLA, Rodrigues MFB, Barbosa IMG, Acioly RF, Carvalho DC, Carvalho RAB et al. Gengivoplastia sem elevação de retalho mucoperiosteal (flapless) assistida por piezocirurgia: relato de caso. Arch Health Invest. 2020;9(3):253-56.

14. Consolaro MF, Sant'ana E, Moura Neto G. Cirurgia piezelétrica ou piezocirurgia em Odontologia: o sonho de todo cirurgião. Rev Dental Press Ortod Ortop Facial. 2007;12(6):17-20. 


\section{CONFLITO DE INTERESSES}

Os autores declaram não haver conflitos de interesse

AUTOR PARA CORRESPONDÊNCIA

Layla Louise de Amorim Rocha

layla2rocha@gmail.com

Submetido em 19/01/2021

Aceito em 20/02/2021 